\title{
Quality of care for obstetric emergencies in 4 general hospitals in Egypt: an observational study of delays in receiving care and blood bank services
}

K.H. Nada, ${ }^{7}$ A.A. Barakat ${ }^{1,2}$ and R.Gipson ${ }^{7}$

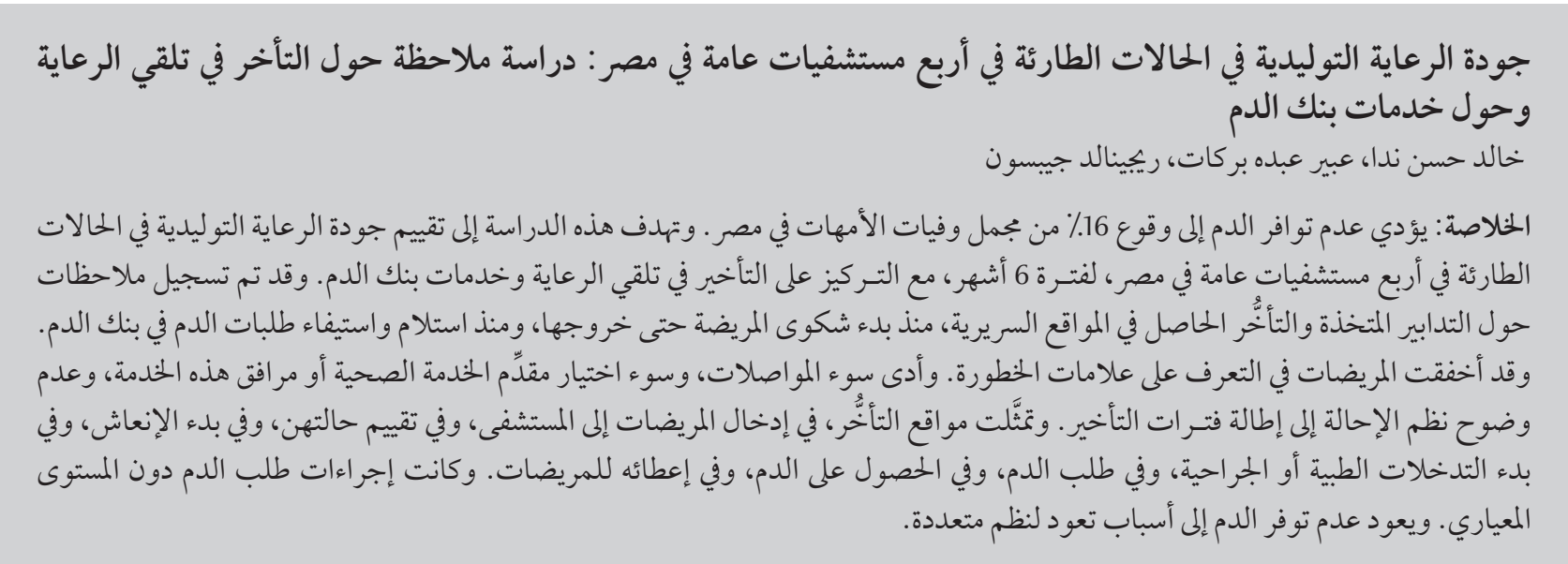

ABSTRACT A lack of available blood contributes to 16\% of all maternal deaths in Egypt. This study aimed to assess the quality of care for obstetric emergencies in 4 general hospitals in Egypt over a 6-month period with the focus on delays in receiving care and blood bank services. Observations were made of the processes and delays in the clinical setting, from the start of each patient's complaint until discharge, and the receipt and filling of orders for blood at the blood bank. Patients failed to recognize danger signs. Lack of transportation, incorrect choice of provider or facility and unclear referral systems added further delays. Delays occurred in hospital admission, assessment of patients, initiation of resuscitation, initiation of medical or surgical interventions, ordering blood, receipt of blood and administration of blood to patients. The blood ordering procedures were substandard. Lack of blood availability had multidisciplinary causes.

Qualité des soins obstétricaux d'urgence dans quatre hôpitaux généraux d'Égypte : étude d'observation sur les retards dans l'administration des soins et la mise en œuvre des services de la banque de sang

RÉSUMÉ Le manque de sang disponible contribue pour $16 \%$ au taux de mortalité maternelle toutes causes confondues en Égypte. La présente étude vise à évaluer la qualité des soins obstétricaux d'urgence dans quatre hôpitaux généraux d'Égypte sur une période de six mois, avec une attention particulière pour les retards dans l'administration des soins et la mise en œuvre des services de la banque de sang. Des observations ont été effectuées sur les processus des soins et les retards dans leur administration en établissements de soins, à partir de l'apparition du motif de consultation des patientes jusqu'à leur sortie de l'établissement de soins, et sur la réception des demandes de sang à la banque de sang et leur exécution. Les patientes n'identifiaient pas les signes de danger. La carence des transports, le choix inadapté d'un prestataire ou d'un établissement et des systèmes peu clairs d'orientation-recours contribuaient à accroître les retards. Des retards se sont produits dans l'admission à l'hôpital, l'examen des patientes et la mise en œuvre d'un protocole de réanimation, mais aussi dans l'instauration d'interventions médicales ou chirurgicales, la demande de sang, la réception et l'administration du sang aux patientes. Les procédures de demande de sang n'étaient pas conformes aux normes. La disponibilité insuffisante du sang avait des causes pluridisciplinaires. 


\section{Introduction}

The World Health Organization (WHO) estimates that 515000 women die each year from pregnancy-related causes, and almost all of these deaths occur in developing countries [1]. In Egypt, despite the decline in maternal mortality from 174/100 000 live births in 1992-93 to 84/100 000 live births in 2000 [2], haemorrhage is stilltheleading direct cause of maternal mortality and is associated with $38 \%$ of all maternal deaths. In the case of either antepartum or postpartum haemorrhage, timely receipt of blood, blood products or volume expanders is required in order to save lives. The lack of available blood has been estimated to contribute to $16 \%$ of all maternal deaths in Egypt [2].

As the time from the onset of haemorrhage to death in pregnant women is often short, WHO recommends that intravenous fluids should be available at all levels of the health care system, and that blood transfusion services should be available at all times in hospitals [3]. This study aimed to assess the quality of care for obstetric emergencies in 4 general hospitals in Egypt with the focus on delays in receiving care and blood bank services.

\section{Methods}

The study used quantitative methods to collect data on emergency obstetric care in 4 general hospitals in Egypt.

\section{Study sites and sample}

The study was conducted in the general hospitals in the 4 governorates where the Egypt Healthy Mother/Healthy Child project was active: Sohag, Assiut, Minya and Giza. All pregnant women presenting to the study hospitals during the observation sessions who needed immediate blood transfusion, or for whom blood transfusion was anticipated based on a clinical assessment, were included in the study.

\section{Data collection}

Data collection started from the beginning of March 2003 and lasted for 6 months. Initially an assessment was made of each facility's blood banks and general blood supply. Then for each woman needing blood transfusion at the study hospitals an analysis was conducted on the different processes involved from the start of the patient's complaint until her discharge.

Three teams of 2 researchers conducted the clinical and blood bank observations in parallel. In each pair one researcher, stationed at the hospital, interviewed patients about events before arrival at the hospital and observed the handling of emergency obstetric cases in the clinical setting. The second researcher, stationed at the hospital blood bank, observed the receipt and filling of orders for blood. All observations in were done in 8-hour shifts covering both day and night and all days of the week including weekends and public holidays.

\section{Tools}

All data collection tools were revised and piloted before data collection started.

\section{Clinical events}

A datasheet was used to record the clinical events for each woman admitted as an obstetric emergency. This included a structured questionnaire to interview the patient or her relatives to document basic demographic data and events occurring before and after arrival at the hospital: the decision to seek care (how long after the problem started was care sought, reasons for choice of provider); and details about transport to the facility (type of transport, time taken).

A checklist was used for direct observation of all activities occurring in the clinical setting: admission procedures (time of admission, time when first examined by physician, time when diagnosis was completed and treatment started); diagnosis (diagnosis recorded by hospital staff on admission, diagnosis recorded by researcher); surgical/ medical interventions (whether needed, whether intervention followed the agreed protocol; what interventions were done, when started, indications for blood transfusion, whether transfusion followed agreed protocols); and laboratory tests (history taken, tests ordered, whether tests were appropriate, when results were received).

All steps related to ordering, preparation and delivery of blood were documented: blood transfusion (whether indicated according to protocol, whether the blood/blood products ordered were the most rational choice for the clinical condition of the patient, and whether the timing and procedures for ordering blood/blood products could have been changed to improve the clinical outcomes). Each order of blood during the data collection period was followed step-by-step and timed.

\section{Blood bank activities}

Another datasheet was used to record blood bank activities. The first section was observations in the blood bank: timing of all stages in the blood orders (order received, action taken, blood sample received, blood group determined; cross-matching completed, blood unit despatched); procedures and personnel involved in making the order; problems with orders and actions taken; types of blood product ordered; and inspection of orders.

The second section was observations of the donation process (if applicable): timing of all stages in donation; why and who requested donation; donor counselling and consent; screening of donors; procedures and personnel involved in the donation process.

\section{Research team}

Data collection required judging the clinical performance of providers, which necessitated a highly trained observation team. Observations in the clinical setting were conducted by assistant lecturers in gynaecology and obstetrics and the blood bank observations were 
conducted by blood bank specialists. To minimize subjectivity on behalf of observers, standard Ministry of Health and Population protocols and guidelines were used as a benchmark to judge clinical and blood bank performance $[4,5]$. The researchers were intensively trained on the objectives, methods and data collection tools, observation methods, case inclusion criteria, guidelines for appropriate use of blood and blood components, standard management of relevant cases, standard information on blood donation, qualitative research, glossary of relevant definitions and logistical arrangements. This was designed to minimize observer bias.

\section{Ethical considerations}

Observers did not interfere in cases even where they were aware of negligence that might result in harm to the patient. This was done for the sake of the validity of research results. However, all remarks were communicated to managers and decision-makers in the hospitals, in order to guide the process of quality improvement inside the hospitals including prevention of recorded delays and harmful practices.

\section{Analysis}

Data validation of the completed forms and the computer data base took place. Epi-Info, version 6.04 was used for data entry and analysis.

\section{Study population}

The total number of women observed in the 4 hospitals was 102. The majority of patients presented to hospital either for delivery $(50 \%)$ or suffering from bleeding (38\%) or other complications.

The mean age of the women was 26.6 [standard deviation (SD) 6.5] years. Nearly three-quarters (73\%) came from rural areas. A high proportion of women were illiterate (63\%) and $13 \%$ could only read and write. Almost all (97\%) were working as housewives, while $36 \%$ of husbands were unskilled manual workers and $52 \%$ skilled manual workers. Participants had a mean parity of 2.3 (SD 0.4).

\section{Delays in attending hospital Choice of provider}

Patients included in the present study lived on average $16.6 \mathrm{~km}$ (SD 4.5) away from the general hospital. Before coming to the hospital, $18 \%$ of the women reported being seen at home by a health care provider. In most cases, the home visits were performed by a daya (traditional birth attendant). A further $31 \%$ of women sought care at the provider's own facility (usually a private clinic) rather then being seen at home; these providers referred them to the general hospital. The remaining majority of the respondents sought care directly at the general hospital.

The most frequent reason mentioned for their choice of any health care provider was the provider's proximity to their home (55\%). Among those who sought care directly at the general hospital, one-third (32\%) went because it was the nearest hospital, and only $6 \%$ went because they believed that they would receive good medical care there.

\section{Patients' transport}

Almost all patients coming to the hospital had made their own transport arrangements. An ambulance was called in only 5 cases; in 4 cases it was called by a relative or neighbour, while in 1 case transport was arranged by a district hospital. Thus of the 23 women referred from a public facility to the general hospital because of an obstetric emergency, all but 1 was left to arrange for her own means of transportation. The most commonly used means of transport were taxi (67\% of cases) or pick-up truck (22\%) (where the patient lies in the open bed of the truck).

\section{Time spent from decision to seek care until arrival at general hospital}

The time reported by patients from recognizing the seriousness ofthe condition until the decision to seek care was only 56 minutes on average. However, when the time spent travelling and attending other providers before finally reaching the general hospital were included, the average time from the decision to seek care until reaching the hospital was 3 hours 25 minutes (Figure 1).

\section{Delays in admission \\ Registration}

Registration of patients on arrival at the hospital required an identification document for the patient or someone accompanying her (for 1 patient the husband had to return home to retrieve it).

\section{Time spent from registration until decision to admit}

Patients arriving at the hospital had to wait on average 20 minutes to be registered by the hospital clerk. The average time taken from registration until a physician examined the patient was 26 minutes. In $21 \%$ of cases the patient arrived at the hospital and was examined first before a decision was taken to admit the patient and register the case. The average total time of both steps was 46 minutes, regardless of which occurred first.

\section{Delays in initial assessment}

Initial assessment and resuscitation

Relevant vital signs were checked in only $62 / 102(62 \%)$ of the study cases. Resuscitation was needed for 61 women (61\%) and was done for 57/61 (93\%) of them. The most common resuscitation measure was initiating a crystalloid intravenous drip (98\%); other measures were emptying the bladder with a Foley catheter $(35 \%)$ or oxygen administration (16\%). Medical supplies needed for resuscitation were generally available, with a few exceptions. Colloids were available in only 1 hospital, but were never observed in use.

\section{Time spent from initial assessment until intervention started}

The time between the physician first examining the patient until a medical or surgical intervention was started was approximately 3 hours. In some of cases 


\section{(39.8\%) the time spent was justified, but in $61 / 102$ cases $(60 \%)$ the long time interval could not be justified on medi- cal grounds by expert obstetricians. \\ Delays in anaesthesia and laboratory investigations}

Anaesthesia was needed in $77 / 102$ cases $(76 \%)$, but was immediately available in less than half of these $(35 / 77$, $45 \%)$. For cases where anaesthesia was needed, but not immediately available, an anaesthetist was called for only $80 \%$ of cases. According to the clinical judgement of the researchers anaesthesia was delayed to the extent that it contributed to patient morbidity in $32 \%$ of the cases where it was needed.

Laboratoryinvestigationswerejudged by the expert obstetricians to be needed for proper clinical decision-making in 69 cases (68\%). However, laboratory investigations were actually performed for just over half of these $(40 / 69,58 \%)$. In $21 \%$ of cases where laboratory investigations were done the investigations were judged by obstetricians to be inappropriate for the patient's condition and in $26 \%$ the results were delayed to the extent of rendering them useless for clinical decision-making or were judged by the expert obstetricians to have delayed the decision-making process itself.

Sonography was available in all 4 hospitals, but in 1 hospital the sonar room was locked after working hours.

\section{Delays in ordering blood for transfusion}

\section{Reservation of blood}

According to the treating physician's diagnosis, 16 cases warranted immediate blood transfusion. Of these cases, immediate transfusion was ordered in 10 cases (63\%), provisional reservation was ordered in $2(13 \%)$ and no blood was reserved in 4 (25\%). A further 85 cases were judged to need provisional reservation of blood. Of these, immediate transfusion was ordered for $6(7 \%)$, provisional reservation was ordered for $38(45 \%)$ and no blood was reserved for 41 (48\%).
Only whole or fresh whole blood was ordered, except in 1 case where packed red blood cells were ordered. A total of 48/56 (86\%) of orders were for only 1 unit of blood. The blood product ordered was the first choice for the clinical condition according to the agreed protocol for $75 \%$ of 56 patients for whom blood was ordered. A history of previous transfusion or transfusion reaction was taken for only 4 of the cases for whom blood was ordered, and it was recorded in the patient's records in 2 of them. In only 7 cases was the reason for transfusion recorded.

A request form was not used for any of cases in which blood was ordered. Instead, the patient's file was sent, usually with a sample in a syringe (64\%). A third of the samples sent had no information written on them at all and most of the remainder had either the first name only or the first and family name written. In a few cases the department name or patient number was written as well. In 1 case the name was written incorrectly, but fortunately the blood bank nurse discovered the mistake. Information on the sample was compared with data in the patient's file in less than a third of the samples sent to the blood bank.

After the order of blood was made, no communication between the treating physicians and the blood bank took place, with 1 exception $(1 / 56)$ (in which the communication was considered inadequate by the researcher).

\section{Time spent from initial assessment until blood ordered}

The average time from the physician first examining the patient until blood was ordered was on average 1 hour 20 minutes for all cases for which blood was requested. Interestingly, the average time between the first examination and the time when blood was ordered was lower for the patients for whom provisional reservation of blood was ordered (1 hour 45 minutes) (Figure 2) than for the patients for whom immediate transfusion of blood was ordered (40 minutes) (Figures 3). 

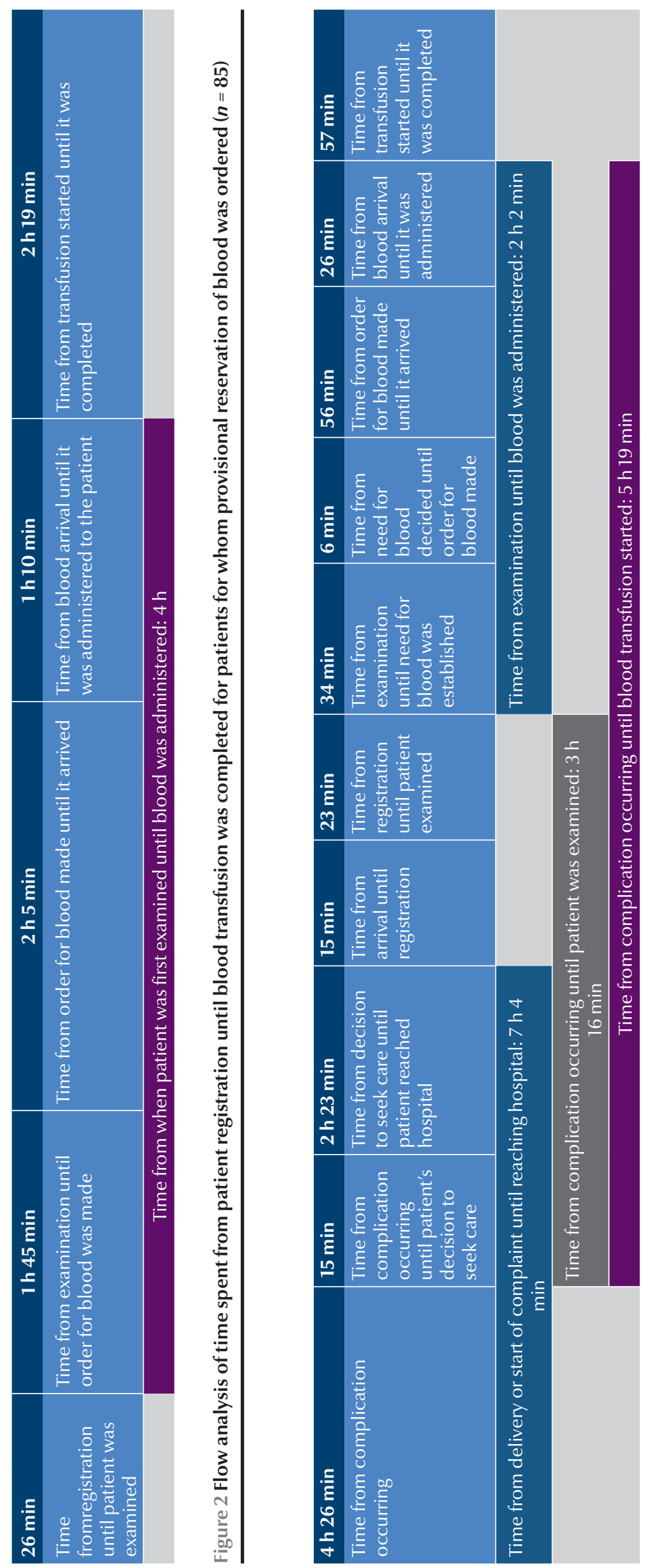

\section{on $\quad$ Time spent from order for blood until blood arrival at the hos- pital}

The average time from ordering blood for immediate transfusion until the blood arrived at the hospital was 50 minutes (Figure 4). In cases of provisional reservation of blood the time interval was 2 hours 5 minutes (Figure 2). This time interval included the time needed for donation by relatives or friends in cases of shortage of the needed blood group (donation took place for 19 patients). It also included the time for serological testing of the donated blood (using the rapid method).

In 1 case blood had to be purchased from the regional blood bank by the patient's relatives (due to the lack of the blood group in the hospital). They brought it in a plastic bag surrounded by ice cubes. It took 1 hour 40 minutes from the time they were asked to buy blood until it was brought to the patient.

\section{Delays in administering blood}

\section{Transfusion of blood}

Of a total of 56 orders, only 22 (40\%) were brought to the clinical setting. Of the received blood $2 / 22$ orders (9\%) were not administered to the patient and were wasted since blood banks follow a non-return policy for blood units dispatched for more than 30 minutes. The blood unit was not properly inspected before administration for any of the cases. The patient's identity was checked against the blood unit in only $40 \%$ of the orders.

Physicians did not record baseline information related to transfusion for any of the patients, except in 1 case. None of the patients were given assurances about the procedure or asked for their con- 


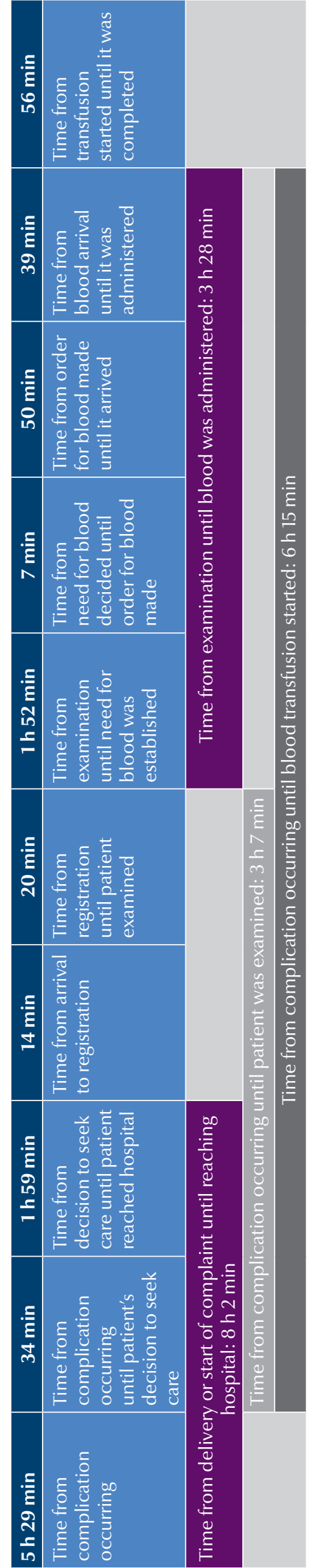

sent, and only $40 \%$ of conscious patients were instructed to report any unusual symptoms.

The administered blood was judged to have been given too rapidly in $90 \%$ of cases, and patient monitoring in the first 15 minutes was only done in 30\% of the cases. There was no system in place to report transfusion reactions in any of the study facilities.

\section{Time spent from blood arrival at hospital until administered to patient}

Once the blood reached the clinical setting, the average time taken until it was administered to patients for whom immediate transfusion was ordered was on average 39 minutes $(n=13)$ (Figure 4$)$. In cases in which blood was only reserved and then later administered to the patient it was 1 hour 10 minutes (Figure 2).

\section{Time spent from start of transfusion until completion}

Once the blood was ready for administration, the time from the start of transfusion till its completion was 56 minutes in cases where immediate blood transfusion took place (Figure 4). In cases where blood was initially reserved, transfusion took longer and was completed in 2 hours 19 minutes (Figure 2).

\section{Outcomes}

One woman died and, in the researcher's judgement, delay in receiving blood was a contributing factor. Thus maternal mortality was the outcome in $1 \%$ of emergency obstetric cases compared with less than $0.2 \%$ in the other admitted patients.

\section{Discussion}

Key factors in the total delay in handling obstetric emergencies in this study was the delay in attending hospital due to women's initial choices of care provider and inadequate transport facilities. While one-third of women (32\%) came directly to the hospital because it was the nearest provider, only $6 \%$ believed they would receive good medical care there. This perception of poor quality of care provided at the general hospitals was likely to be a factor in the delay in seeking care. This acts both directly through prolonging the time until the decision to seek care is taken and indirectly through the decision to go to other providers who are not equipped to handle these emergencies. Barnes-Jasiah et al. identified confidence in the medical services as a crucial factor in delaying, or never making, the decision to seek care [6]. Also, a shortage in obstetricians can affect birth outcomes dramatically. In rural Washington women from "high-outflow" communities, where women deliver far from local hospitals due to shortage in local providers, had a higher rate of complicated deliveries than women from communities where most patients delivered in the local hospital [7]. Another major concern is that patients coming to the hospital nearly always made their own transport arrangements. In Tanzania participatory community plans to solve transport-related delays in handling maternal emergencies were developed in 50 villages and showed some success in collecting funds for and organizing better transport systems [8].

The next component of the total delay in emergency obstetric care was the delay in initiating care. The average total time for both registering then first examining the patient was 46 minutes. Delayed anaesthesia, delayed laboratory results and a locked sonar room contributed to patient 
morbidity. This is not unique to developing countries; for example, a national survey in England and Wales found that anaesthesia was delayed during obstetric emergencies in $29 \%$ of cases [9].

Deficiencies in quality of care were not judged by our researchers to be related to lack of resources. Research has indicated that even countries with an annual per capita income of only US $\$ 200$ have succeeded in substantially reducing maternal mortality. Maternal mortality is more closely linked to poor organization of services than to a lack of national resources [10]. For example, the dramatic decline in the incidence of haemorrhage as a cause of maternal mortality in Commonwealth countries between 1954 and 1985 appears to have been due to both legislative actions and to improvements in medical practice such as licensing of maternity services and blood banks [11].

Communication between the treating physician and the blood bank never took place, with 1 exception. Samples were sent without information, with incomplete information or with information on the sample and "request document" not cross-checked. These factors exponentially increase the risk of transfusing incompatible blood. Research shows that the majority of errors related to transfusions occur outside the blood bank [12], which was also the case in our study. The seriousness of such errors should not be underestimated. Presently in developed countries, transfusion errors are a greater source of morbidity and mortality than transfusion-transmitted infections [13].

Our observations showed that practices related to blood transfusion were poor; the blood units were not properly inspected before administration in any of the cases and the patient's identity was checked against the blood unit in only $40 \%$ of the cases. Studies show that patient and blood unit identification are not always done to the highest standard, even in developed countries. In a study in the US, completion of 4 specific components of the identification process was achieved in $62.3 \%$ of cases, whereas completion of all steps was achieved in $25.4 \%$ of cases [14].

WHO has frequently emphasized the need for a basic infrastructure and resources for the establishment of a plasma fractionation in developing countries as a tool for radical upgrading of countries' blood transfusion systems. Rapid transfusion and lack of monitoring before and after commencement of transfusion are life-threatening issues since the most serious transfusion reactions occur during that time [15].

\section{Conclusions}

In the current study, the delay in receiving blood for transfusion was a key issue in the quality of care of women with obstetric emergencies. The problem is multidisciplinary. Availability of blood and delays in delivering it are not constrained by the availability of resources, which were judged to be available to an acceptable level in all study hospitals. Controlling this problem in Egypt would require better organization and practices in the blood bank service, improved clinical performance within the general hospitals and better management of patient admissions and referrals. Interventions should also be directed towards women and health care decision-makers within households to help them to recognize the warning symptoms of obstetric emergencies and to appreciate the need to deal with these immediately.

\section{References}

1. Mavalankar DV, Rosenfield A. Maternal mortality in resourcepoor settings: policy barriers to care. American Journal of Public Health, 2005, 95:200-203.

2. National maternal mortality study, 2000. Cairo, Egypt, Ministry of Health and Population, 2001.

3. Maternal Health and Safe Motherhood Programme, Division of Family Health. The mother-baby package. Implementing safe motherhood in countries: practical guide. Geneva, World Health Organization, 1996.

4. Blood Transfusion Service. Part 1: Guidelines for appropriate use of blood and blood components, 1st ed. Cairo, Egypt, Ministry of Health and Population, 1999.

5. Essential obstetric care: protocols for physicians. Cairo, Egypt, Ministry of Health and Population, 2001.

6. Barnes-Josiah D, Myntti C, Augustin A. The "three delays" as a framework for examining maternal mortality in Haiti. Social Science \& Medicine, 1998, 46:981-993.

7. Nesbitt TS et al. Access to obstetric care in rural areas: effect on birth outcomes. American Journal of Public Health, 1990, 80:814-818.

8. Schmid T et al. Transportation for maternal emergencies in Tanzania: empowering communities through participatory problem solving. American Journal of Public Health, 2001, 91:1589-1590.
9. Qureshi AM, Stevens M, Plaat F. Survey of anesthetic support staff in obstetric units in England and Wales. Anaesthesia, 2003, 58:578-582.

10. Alihonou E. Africa: time for action. Planned Parenthood Challenges, 1998, (1):15.

11. Sachs BP et al. Hemorrhage, infection, toxemia, and cardiac disease, 1954-85: causes for their declining role in maternal mortality. American Journal of Public Health, 1988, 78:671-675.

12. Sharma RR, Kumar S, Agnihotri SK. Sources of preventable errors related to transfusion. Vox Sanguinis, 2001, 81:37-41.

13. Allain JP. Risques transfusionnels d'hier et d'aujourd'hui [Transfusion risks of yesterday and of today]. Transfusion Clinique et Biologique, 2003, 10:1-5.

14. Novis DA et al. Audit of transfusion procedures in 660 hospitals. A College of American Pathologists Q-probes study of patient identification and vital sign monitoring frequencies in 16494 transfusions. Archives of Pathology and Laboratory Medicine, 2003, 127:541-548.

15. Fereydoun A, Thierry B, El-Nageh M. Plasma fractionation programmes for developing countries: technical aspects and infrastructural requirements. Alexandria, Egypt, World Health Organization. Regional Office for the Eastern Mediterranean, 1999 (Eastern Mediterranean Series, No. 22). 